\title{
Dense Plasma Torus in the GPS Galaxy NGC 1052
}

\author{
Seiji Kameno ${ }^{1}$, Makoto Inoue ${ }^{1}$, Kiyoaki Wajima ${ }^{1}$, \\ Satoko Sawada-Satoh ${ }^{2}$ and Zhi-Qiang Shen ${ }^{2, *}$ \\ ${ }^{1}$ National Astronomical Observatory of Japan, 2-21-1 Osawa, Mitaka Tokyo, Japan, 181-8588 \\ kameno@hotaka.mtk.nao.ac.jp \\ inoue@nao.ac.jp \\ kiyoaki@hotaka.mtk.nao.ac.jp \\ ${ }^{2}$ The Institute of Space and Astronautical Science, 3-1-1 Yoshinodai, Sagamihara \\ Kanagawa, Japan, 229-8510 \\ satoko@vsop.isas.ac.jp \\ zshen@asiaa.sinica.edu.tw
}

Received 2002 July 30, accepted 2003 February 13

\begin{abstract}
We report results from nearly simultaneous pentachromatic VLBI observations towards a nearby GPS galaxy NGC 1052. The observations at 1.6 and $4.8 \mathrm{GHz}$ with the VSOP, and at 2.3, 8.4, and $15.4 \mathrm{GHz}$ with VLBA, provide linear resolutions of $\sim 0.1 \mathrm{pc}$. Convex spectra of a double-sided jet imply that synchrotron emission is obscured through foreground cold dense plasma, in terms of free-free absorption (FFA). We found a central condensation of the plasma which covers about 0.1 and $1 \mathrm{pc}$ of the approaching and receding jets, respectively. A simple model with a geometrically thick plasma torus perpendicular to the jets is established to explain the asymmetric distribution of FFA opacities.
\end{abstract}

Keywords: galaxies: active — galaxies: individual (NGC 1052, 0238-084) — galaxies: nuclei — galaxies: jets — radio continuum: galaxies — techniques: high angular resolution

\section{Introduction}

Gigahertz peaked spectrum (GPS) sources show a convex radio spectrum peaked at $\mathrm{GHz}$ frequencies, as they are named, and a compact size of $\lesssim 1 \mathrm{kpc}$ (e.g. O'Dea 1998). The spectral shape indicates that a power-law spectrum of optically thin synchrotron radiation is affected by a lowfrequency cutoff. It is a controversial issue as to what is the origin of the low-frequency cutoff: synchrotron selfabsorption (SSA) or free-free absorption (FFA).

Tight correlation between the peak frequency and the overall linear size (O’Dea \& Baum 1997), and correlation between the equipartition size and the overall linear size (Snellen et al. 2000) indicate that SSA controls the the peak frequency, at which the equipartition size is derived by Scott \& Readhead (1977).

Bicknell, Dopita, \& O'Dea (1997), on the other hand, pointed out that FFA through ionised gas surrounding radio lobes can also produce the correlation between the peak frequency and the overall size. Evidence for FFA was found via multifrequency VLBI observations in particular GPS sources: 1946+708 (Peck, Taylor, \& Conway 1999), OQ 208 (Kameno et al. 2000; Xiang et al. 2002), 0108+388 (Marr, Taylor, \& Crawford 2001), and NGC 1052 (Kameno et al. 2001; Kadler et al. 2003).

We propose a clear method to discriminate between the absorption mechanisms. In the case of SSA, the absorber is the synchrotron emitter itself such as jets or lobes.

\footnotetext{
* Present address: Academia Sinica Institute of Astronomy and Astrophysics, Department of Physics, National Taiwan University, No. 1, Roosevelt Road, Sec. 4, Taipei 106, Taiwan.
}

Relativistic speeds of the emitter will cause significant Doppler shift of the peak frequency; approaching and receding jets will show blue- and redshifted spectra, respectively. Hence, we expect a higher peak frequency for an approaching jet. In the case of FFA, ambient cold dense plasma is an absorber whose speed is non-relativistic. We expect greater opacity towards receding jets to produce a higher peak frequency. This picture was drawn towards some radio galaxies such as 3C 84 (Vermeulen, Readhead, \& Backer 1994; Walker, Romney, \& Benson 1994; Walker et al. 2000), Cen A (Jones et al. 1996; Tingay \& Murphy 2001), NGC 4261 (Jones et al. 2000, 2001), and NGC 6251 (Sudou et al. 2000). The opposite spectral behaviour between SSA and FFA allows us to discriminate the absorption mechanisms by measurements of the peak frequencies towards approaching and receding jets. Multifrequency observations towards GPS sources with double-sided jets are relevant to this issue.

We chose the nearest GPS source, NGC 1052, for this study. The redshift of this galaxy, $z=0.0049$ (Knapp et al. 1978), corresponds to a distance of $20 \mathrm{Mpc}$, if we assume $H_{0}=75 \mathrm{~km} \mathrm{~s}^{-1} \mathrm{Mpc}^{-1}$ and $q_{0}=0.5$, and 1 milliarcsec (mas) corresponds to $0.1 \mathrm{pc}$. This object has parsec-scale twin jets in $\mathrm{PA} \sim 65^{\circ}$ (Claussen et al. 1998), so that it is suitable for the discrimination of the absorbing mechanism. Their observations also revealed that $\mathrm{H}_{2} \mathrm{O}$ maser spots are distributed along the western jet with a velocity gradient. Although the radio emission is dominated by a compact extent of $\lesssim 1^{\prime \prime}$ (Wrobel 1984), two lobes of total extent $\sim 2.8 \mathrm{kpc}$ straddle the central component and are aligned in PA $\sim 95^{\circ}$ (Jones, Wrobel, \& Shaffer 1984; 

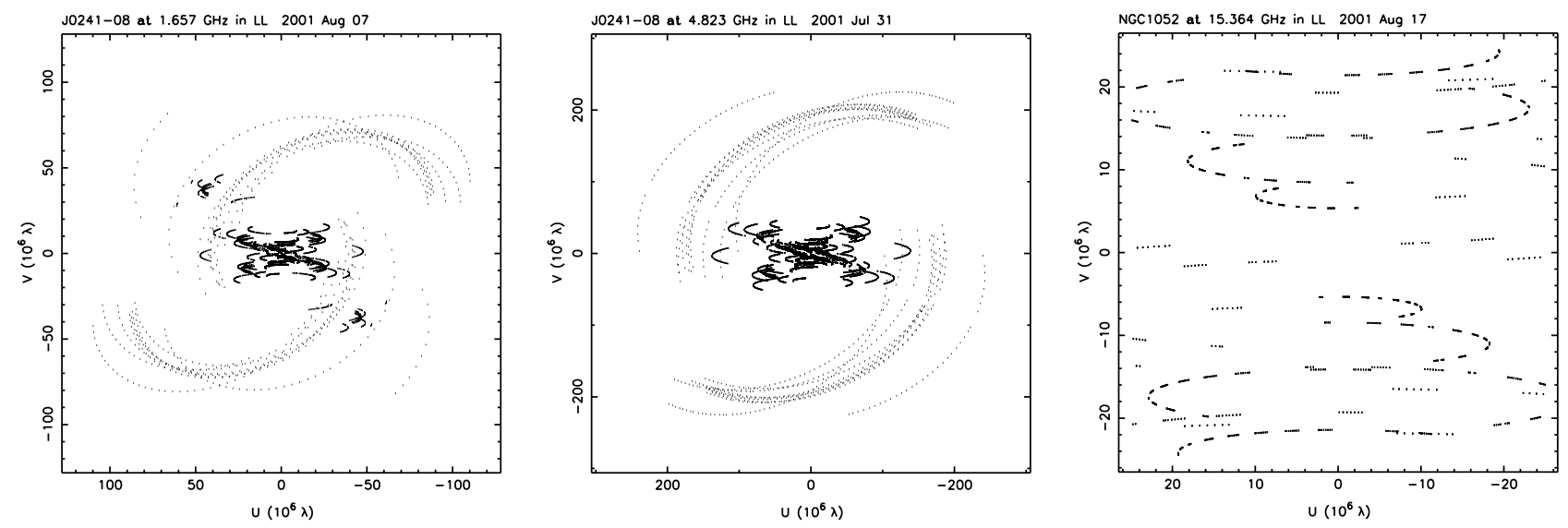

Figure 1 Distribution of visibilities in spatial frequencies ( $u v$ coverage) at 1.6 (left) and 4.8 (middle) GHz. Inner dense and outer sparse sets are attributed to the ground-ground and space-ground baselines, respectively. The ground-ground array at $4.8 \mathrm{GHz}$ consists of the $10 \mathrm{VLBA}$ stations, while the ATCA operated at $1.6 \mathrm{GHz}$. The $u v$ coverages at $2.3,8.4$, and $15.4 \mathrm{GHz}$ are similar to the ground-ground $u v$ coverage at $4.8 \mathrm{GHz}$, scaled by frequencies. Right: The inner $25 \mathrm{M} \lambda$ area of the $u v$ coverage at $15.4 \mathrm{GHz}$. The lack of visibilities within $5 \mathrm{M} \lambda$ will cause uncertainties in any structure larger than 20 mas.

Wrobel 1984; Wrobel \& Heeschen 1984). This indicates that NGC 1052 belongs to the $9-15 \%$ minority of GPS sources with an extended structure (Stanghellini et al. 1998).

Our previous trichromatic VLBA observations for the first time clarified the jet geometry and presence of a dense plasma torus that is responsible for FFA (Kameno et al. 2001). The eastern and western sides of the jet are approaching and receding with the velocity of $\beta=0.25$ at the viewing angle of $50^{\circ}$ (Kameno et al. 2001). The western receding jet is obscured at lower frequencies, suggesting that a dense plasma torus covers the receding jet to produce FFA (Kameno et al. 2001; Kadler et al. 2003; Vermeulen et al. 2003). Although the conclusion was well justified, three frequencies were not sufficient to obtain a confident fit for the spectrum.

In this paper, we report new pentachromatic VLBI observations of NGC 1052 using the VSOP (Hirabayashi et al. 1998) and the VLBA.

\section{Observations and Results}

All pentachromatic observations have been carried out within 18 days during July 31 through August 31, 2001. We used the space-VLBI (VSOP) array at 1.6 and $4.8 \mathrm{GHz}$, and VLBA at 2.3, 8.4, and 15.4 GHz. This combination provides comparable resolutions of $\sim 1$ mas through an order of magnitude in frequency except at $2.3 \mathrm{GHz}$.

Observations at $2.3,8.4$, and $15.4 \mathrm{GHz}$ using VLBA, coded BK084, were done on August 7, 2001. We used the dual-frequency $2.3 / 8.4$ and the $15.4 \mathrm{GHz}$ receiving systems, switching by 22-min scan cycles. Total integration time consists of 10 and 11 scans for $2.3 / 8.4$ and $15.4 \mathrm{GHz}$, respectively. Four $8 \mathrm{MHz}$ baseband converters (BBCs) are allocated for $15.4 \mathrm{GHz}$, while two BBCs are used for 2.3 and $8.4 \mathrm{GHz}$, respectively. Fringes were significantly detected with all valid baselines and scans.
Space-VLBI(VSOP) observations at $1.6 \mathrm{GHz}$ (W513A) and $4.8 \mathrm{GHz}$ (W513B) were executed on August 7 and July 31, 2001, respectively. The spacecraft HALCA was linked to two tracking stations during each observation: Green Bank and Tidbinbilla for W513A, and Green Bank and Usuda for W513B. Though fringes within the ground array were strong enough, those in space baselines were marginal at $4.8 \mathrm{GHz}$. While visibilities with the tracking pass using Green Bank were available, we missed them in the Usuda tracking pass. Fringes at $1.6 \mathrm{GHz}$ were entirely detected within ground and space baselines. Figure 1 shows distributions of visibilities in spatial frequencies ( $u v$ coverages) at 1.6 and $4.8 \mathrm{GHz}$. Inner dense and outer sparse sets of visibilities are attributed to the ground-ground and space-ground baselines. At $2.3,8.4$, and $15.4 \mathrm{GHz}$, the $u v$ coverages are similar to the ground-ground $u v$ coverage at $4.8 \mathrm{GHz}$, scaled by frequencies.

Fringe fitting, passband correction, and a priori amplitude calibrations were made using the NRAO AIPS. Image synthesis and self-calibration were processed with Difmap. The gscale process was applied to match visibility amplitudes among different BBCs and baselines. Image performance is summarised in Table 1 .

Differences in the synthesised beams are due to different sets of spatial frequencies ( $u v$ coverage). To compare intensities between five images, we then made images with the identical restoring beam of $1.9 \times 1.4$ mas in $\mathrm{PA}=0^{\circ}$. The restoring beam size is chosen to represent all synthesised beams except that at $2.3 \mathrm{GHz}$. This treatment is roughly identical with equalising the $u v$ coverages by adjusting the weighting functions of visibilities.

Another concern arises due to the lack of short-spacing baselines. It will cause missing flux for extended structures. This problem is more serious at higher frequencies. The shortest $u v$ radius $\left(=\sqrt{u^{2}+v^{2}}\right)$ at $15.4 \mathrm{GHz}$ is $5 \mathrm{M} \lambda$, as shown in Figure 1, which results in uncertainties in the 
Table 1. Image performance

\begin{tabular}{|c|c|c|c|c|c|}
\hline \multirow{2}{*}{$\begin{array}{l}\text { Frequency } \\
(\mathrm{GHz})\end{array}$} & \multicolumn{3}{|c|}{ Beam } & \multirow{2}{*}{$\begin{array}{c}\text { Image rms } \\
\left(\mathrm{mJy} \mathrm{beam}^{-1}\right)\end{array}$} & \multirow[t]{2}{*}{ Telescopes } \\
\hline & Major (mas) & Minor (mas) & PA (deg.) & & \\
\hline 1.6 & 1.75 & 1.39 & 31.3 & 1.2 & HALCA, ATCA, and VLBA \\
\hline 2.3 & 6.34 & 2.58 & -5.8 & 0.5 & VLBA \\
\hline 4.8 & 0.78 & 0.67 & -78.7 & 1.5 & HALCA and VLBA \\
\hline 8.4 & 1.86 & 0.77 & -2.2 & 0.4 & VLBA \\
\hline 15.4 & 1.09 & 0.42 & -7.8 & 0.4 & VLBA \\
\hline
\end{tabular}
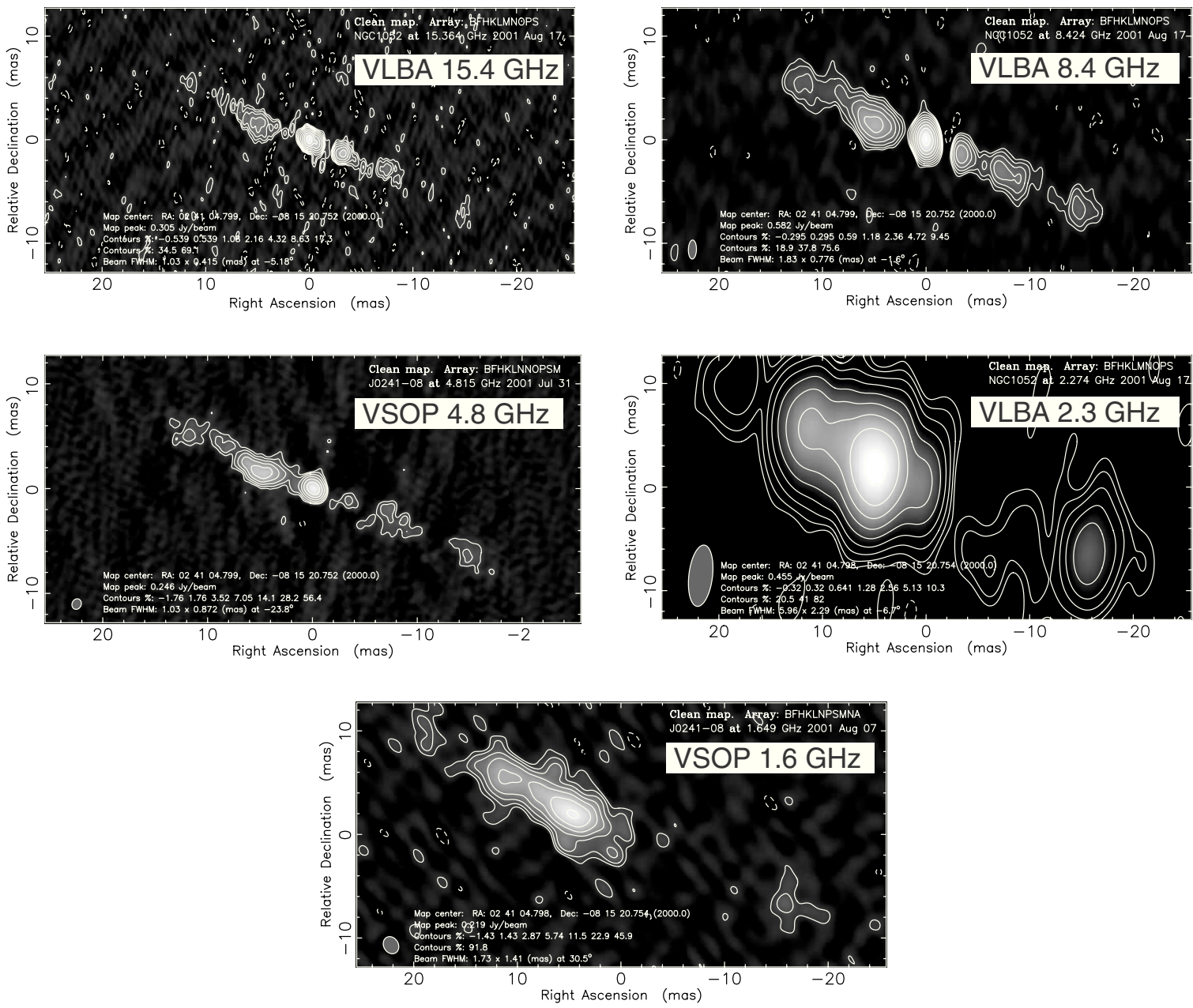

Figure 2 Pentachromatic images of NGC 1052. Image performance is listed in Table 2. Contours start at \pm 3 times the rms level and increase by factors of 2 . Synthesised beams are shown at left bottom corner in each image.

determination of structures larger than $\sim 20$ mas. Nevertheless, comparison in the central 10 mas region across five frequencies will not be seriously affected by such a hole in the $u v$ sampling. We took no further consideration about mismatch of $u v$ coverages.

Not only the image rms in Table 1 but also errors in the amplitude calibration should be accounted for in the accuracy of flux densities. We estimated the accuracy of the amplitudes from standard deviations of gain correction factors through the gscale process in Difmap. At every frequency, the standard deviation of the gain correction factors among 10-12 antennas was $2-5 \%$. Thus, we added the root-sum-square of $5 \%$ of the intensity at each pixel and the image rms to the uncertainty of the intensity.

Figure 2 shows images at five frequencies. Each image includes an uncertainty in absolute position through the self-calibration process. To register the images at different frequencies, we picked up six distinct components: $\mathrm{A} 1, \mathrm{~A} 2$, and $\mathrm{A} 3$ in the approaching jet, and R1, R2, and $\mathrm{R} 3$ in the receding jet. Peak intensities of these components are listed in Table 2. We then measured the positions $\left(\xi_{\mathrm{k}}^{v}, \eta_{\mathrm{k}}^{v}\right)$ of them with respect to a tentative origin of each 
Table 2. Peak intensity of components*

\begin{tabular}{lccccc}
\hline Component & \multicolumn{5}{c}{ Peak brightness $\left(\mathrm{mJy}_{\text {beam }}{ }^{-1}\right)$} \\
\cline { 2 - 5 } & $1.6 \mathrm{GHz}$ & $2.3 \mathrm{GHz}$ & $4.8 \mathrm{GHz}$ & $8.4 \mathrm{GHz}$ & $15.4 \mathrm{GHz}$ \\
\hline A1 & $58.3 \pm 3.0$ & $54.2 \pm 3.3$ & $21.5 \pm 1.8$ & $14.0 \pm 0.9$ & $8.9 \pm 0.7$ \\
A2 & $212.6 \pm 10.6$ & $287.3 \pm 14.5$ & $185.8 \pm 9.4$ & $125.7 \pm 6.3$ & $65.5 \pm 3.3$ \\
A3 & $10.5 \pm 0.7$ & $37.5 \pm 2.6$ & $350.1 \pm 17.6$ & $715.7 \pm 36.0$ & $578.7 \pm 28.9$ \\
R1 & $4.3 \pm 0.6$ & $19.9 \pm 2.1$ & $20.7 \pm 1.8$ & $14.8 \pm 0.9$ & $7.5 \pm 0.7$ \\
R2 & $<0.5$ & $<1.9$ & $24.4 \pm 1.9$ & $31.4 \pm 1.7$ & $26.2 \pm 1.4$ \\
R3 & $1.3 \pm 0.5$ & $<1.9$ & $17.6 \pm 1.7$ & $58.7 \pm 3.0$ & $146.9 \pm 7.4$ \\
\hline
\end{tabular}

* Peak intensity values of components in the images with the identical restoring beam of $1.9 \times 1.4$ mas are listed. Uncertainties include the amplitude calibration error of 5\% and the image rms.

Table 3. Accuracy of image registration

\begin{tabular}{lcc}
\hline $\begin{array}{l}\text { Frequency } \\
(\mathrm{GHz})\end{array}$ & $\begin{array}{c}\text { RA } \\
(\mathrm{mas})\end{array}$ & $\begin{array}{c}\mathrm{dec} \\
(\mathrm{mas})\end{array}$ \\
\hline 15.4 & 0.30 & 0.29 \\
8.4 & 0.21 & 0.31 \\
4.8 & - & - \\
2.3 & 2.80 & 0.75 \\
1.6 & 0.94 & 0.43 \\
\hline
\end{tabular}

image. Here $\mathrm{k}$ and $v$ stand for indices of components and frequency, respectively. We then derived relative offsets $\left(\delta \xi^{v}, \delta \eta^{v}\right)$ to minimise the positional residuals $\chi_{v}^{2}$ defined as

$$
\chi_{v}^{2}=\sum_{\mathrm{k}}\left[\frac{\left(\xi_{\mathrm{k}}^{v}-\delta \xi^{\nu}-\xi_{\mathrm{k}}^{\nu_{0}}\right)^{2}}{\sigma_{\xi_{\mathrm{k}}^{v}}^{2}+\sigma_{\xi_{\mathrm{k}}^{\nu_{0}}}^{2}}+\frac{\left(\eta_{\mathrm{k}}^{v}-\delta \eta^{v}-\eta_{\mathrm{k}}^{\nu_{0}}\right)^{2}}{\sigma_{\eta_{\mathrm{k}}^{v}}^{2}+\sigma_{\eta_{\mathrm{k}}^{\nu_{0}}}^{2}}\right],
$$

where $\sigma_{\xi_{\mathrm{k}}^{\nu}}$ and $\sigma_{\eta_{\mathrm{k}}^{\nu}}$ are standard positional errors of component $\mathrm{k}$ at frequency $\nu$, and $\nu_{0}$ is the frequency of the reference image. We take $v_{0}=4.8 \mathrm{GHz}$, where the frequency is in the middle of our observing range, and where the synthesised beam is the sharpest. Standard errors of the registration are listed in Table 3.

\section{Discussion}

First of all, we aim at clarifying the absorbing mechanism. Figure 3 shows continuum spectra of six components. Receding components R1, R2, and R3 show sharp cutoff at the low-frequency ends. The spectral index of R1 between 1.6 and $2.3 \mathrm{GHz}$ is $\alpha_{1.6}^{2.3}=4.76 \pm 0.54$. Here we define the spectral index $\alpha$ as $S_{v} \propto v^{+\alpha}$, where $S_{v}$ is the flux density and $v$ is the frequency. The spectral index exceeds the maximum attainable spectral index of 2.5 by SSA. For component R3, $\alpha_{1.6}^{4.8}=2.43 \pm 0.38$ is also close to the limit by SSA. Componenent R2 is not detected below $2.3 \mathrm{GHz}$ due to the sharp cutoff in its spectrum. If we use three times the image rms as the upper limit of the flux density, a lower limit of spectral index $\alpha_{1.6}^{4.8}>2.56$ is obtained. The brightest approaching component, A3, shows $\alpha_{1.6}^{4.8}=3.28 \pm 0.27$. These steeply rising spectra are significant evidence for the presence of FFA, which can produce $\alpha>2.5$.

Component A1 shows a single power-law spectrum with $\alpha=-1.1$, whose spectral peak must be at $<1.6 \mathrm{GHz}$, while the spectral peak of its counter-component (corresponding two-sided jet at the similar location to the centre) R1 is between 2.3 and $4.8 \mathrm{GHz}$. Components A2 and A3 mark the spectral peaks at $\sim 2.3$ and $\sim 8.4 \mathrm{GHz}$, respectively, whose counter-components $\mathrm{R} 2$ and $\mathrm{R} 3$ show spectral peaks at $\sim 8.4$ and $>15.4 \mathrm{GHz}$. Every approaching component shows a lower peak frequency than its counterpart does. As is mentioned in the introduction, this clearly indicates that the receding jet is obscured by an external absorber, say, FFA.

To illustrate the spatial distribution of opacity, we applied a spectral fit for the FFA model

$$
S_{v}=S_{0} v^{\alpha_{0}} \exp \left(-\tau_{0} v^{-2.1}\right),
$$

pixel by pixel. Here, $S_{v}$ is the observed flux density at the frequency $v$ in $\mathrm{GHz}, \alpha_{0}$ is the intrinsic spectral index, $S_{0}$ is the absorption-corrected flux density at $1 \mathrm{GHz}$, and $\tau_{0}$ is the FFA coefficient. We fixed $\alpha_{0}$ to -1.1 , which is obtained from the spectral index of component A1. Figure 4 shows the spatial distribution of $\tau_{0}$ and its profile along the jet. The maximum value of $\tau_{0} \sim 300$ is consistent with the results by Kameno et al. (2001). The profile tails over $\sim 1$ pc towards the western receding jet, while it rapidly decreases towards the eastern approaching jet. This asymmetric profile is again consistent with Kameno et al. (2001), with more detailed structure from the high resolution of the VSOP.

Since the opacity coefficient, $\tau_{0}$, is related to the electron density, $n_{\mathrm{e}}$, and temperature, $T_{\mathrm{e}}$, along the line of sight as

$$
\tau_{0}=0.46 \int_{\mathrm{LOS}} n_{\mathrm{e}}^{2} T_{\mathrm{e}}^{-3 / 2} \mathrm{~d} L,
$$

it will be an indicator of cold dense plasma. Here, the integration stands for path length along the line of sight in units of pc. When the absorber is homogeneous, $\tau_{0}$ is proportional to the path length through the ionised region. A simple model to explain the opacity profile is shown in 

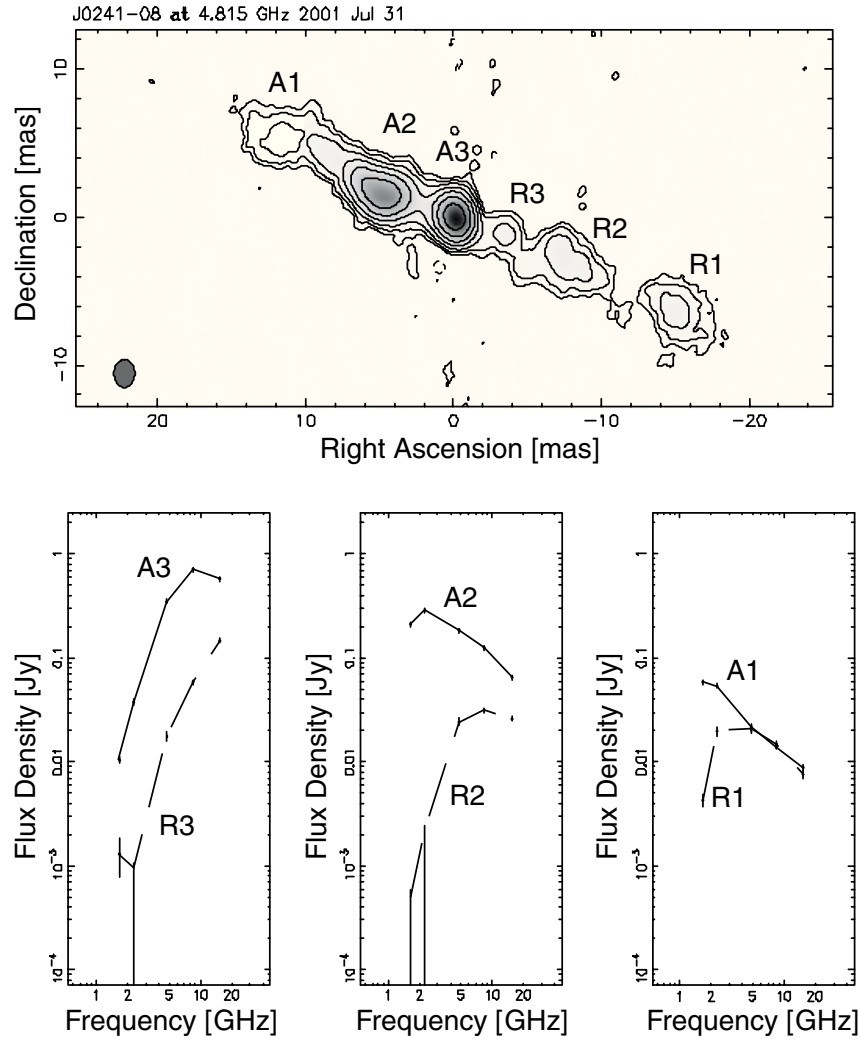

Figure 3 Top: Total intensity map at $4.8 \mathrm{GHz}$ restored by an elliptical Gaussian whose FWHM is $1.9 \times$ 1.4 mas in PA $=0^{\circ}$. Contours start at $\pm 3 \sigma$ level and increase by factors of 2 , where $\sigma=1.13 \mathrm{mJy} \mathrm{beam}^{-1}$. Approaching and receding components are marked as $\mathrm{A}_{k}$ and $\mathrm{R}_{k}$, where $k=1,2$, and 3. Bottom: Spectra of components. Values are listed in Table 2.
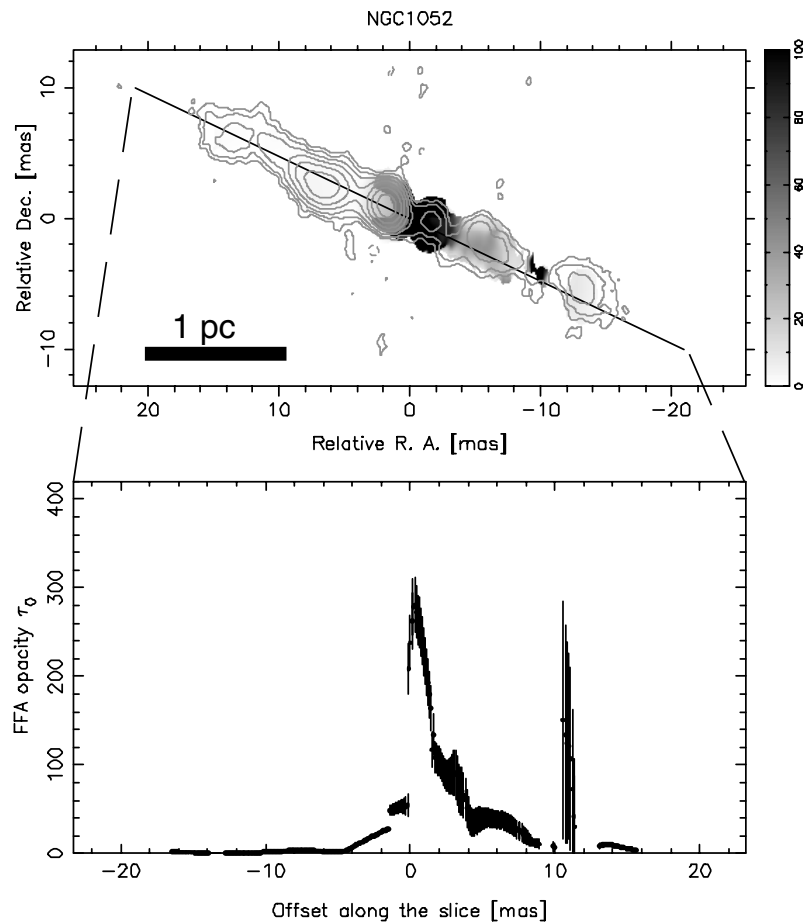

Figure 4 Top: Spatial distribution of FFA opacity $\tau_{0}$ in grey scale overlaid on the total intensity map at $4.8 \mathrm{GHz}$ in contour. Bottom: Profile of the FFA opacity along the jet. Errors are derived from the nonlinear spectral fit using equation (2).

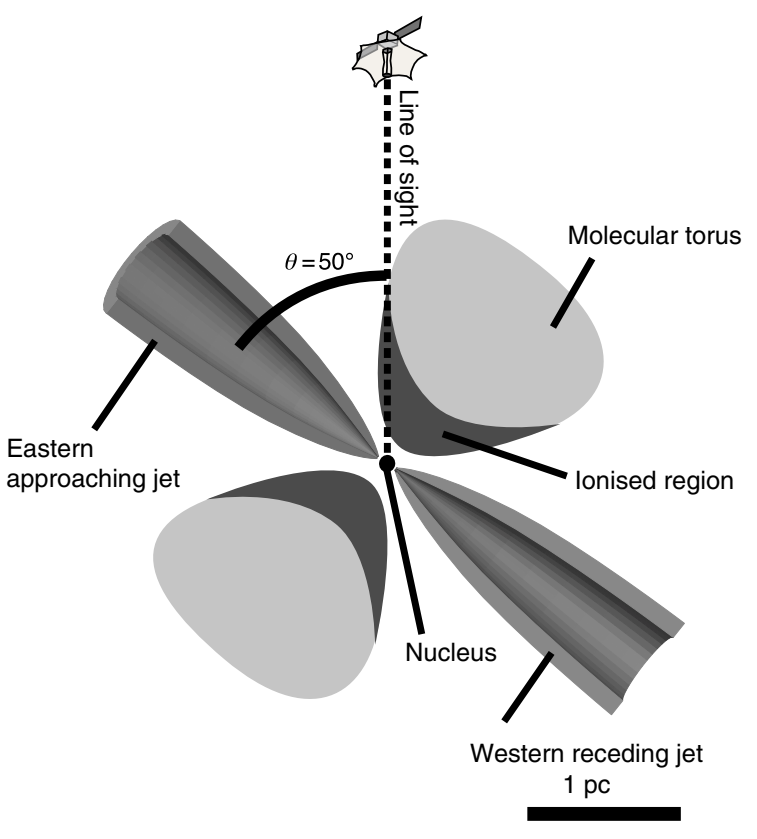

Figure 5 Schematic diagram of NGC 1052. The double-sided jet is inclined by $50^{\circ}$ with respect to the line of sight. A geometrically thick molecular torus is perpendicular to the jet, and its inner surface is illuminated by ionising photons from the nucleus. The ionised region obscures radio emission from the jet, in terms of free-free absorption. The long path length through the ionised region towards the nucleus results in a large opacity, as is shown in Figure 4. 
Figure 5 with a geometrically thick torus perpendicular to the jets. The inner surface of the torus is photoionised by illumination of ionising photons from the nucleus, while the outer region remains as neutral gas or dust. The path length through the ionised region can produce the asymmetric profile of the opacity, when we look from the viewing angle of $50^{\circ}$.

We can estimate the physical condition of the absorber, if we assume the torus model. The maximum value of $\tau_{0} \sim 300$ towards the nucleus gives

$$
n_{\mathrm{e}}^{2} T_{\mathrm{e}}^{-3 / 2}=650,
$$

when the path length is the same as the width of the absorbed area covering the receding jet, say, $L \sim 1 \mathrm{pc}$. The ionising condition of $T_{\mathrm{e}} \geq 10^{4} \mathrm{~K}$ gives the lower limit of $n_{\mathrm{e}} \geq 2.5 \times 10^{4} \mathrm{~cm}^{-3}$. The upper limit of $T_{\mathrm{e}}$ can be given by the absence of significant free-free emission below the brightness temperature of $T_{\mathrm{b}} \leq 4.9 \times 10^{6} \mathrm{~K}$ at $15.4 \mathrm{GHz}$ or $T_{\mathrm{b}} \leq 5.4 \times 10^{6} \mathrm{~K}$ at $8.4 \mathrm{GHz}$. Since the torus is optically thick, in terms of bremsstrahlung, we have $T_{\mathrm{e}} \sim T_{\mathrm{b}} \leq 4.9 \times 10^{6} \mathrm{~K}$ and then $n_{\mathrm{e}} \leq 2.6 \times 10^{6} \mathrm{~cm}^{-3}$. This evaluation indicates that the FFA absorber consists of cold $\left(10^{4} \leq T_{\mathrm{e}} \leq 4.9 \times 10^{6} \mathrm{~K}\right)$ and dense $\left(2.5 \times 10^{4} \leq n_{\mathrm{e}} \leq 2.6 \times\right.$ $10^{6} \mathrm{~cm}^{-3}$ ) plasma. The average value of the electron density derives the electron column density across the plasma torus ( $L \sim 1 \mathrm{pc}$ ),

$$
n_{\mathrm{e}} L=0.8 \times 10^{23}\left(\frac{T_{\mathrm{e}}}{10^{4} \mathrm{~K}}\right)^{3 / 4} \mathrm{~cm}^{-2} .
$$

This electron column density is comparable to the atomic column density of $\sim 10^{23} \mathrm{~cm}^{-2}$ measured by ROSAT and ASCA X-ray observations (Guainazzi \& Antonelli 1999).

We mention that the location of water maser spots (Claussen et al. 1998) coincides with where the FFA opacity is large. The torus model explains the results of the water masers: the $\mathrm{H}_{2} \mathrm{O}$ molecules can survive inside the torus, shielded against exposure to the nucleus. The seed photons from the receding jet are amplified by excited molecules in the torus. Amplification does not work towards the approaching jet, because the torus is not in front of the jet which supplies continuum seed photons. Absence of masers towards the eastern jet can be understood by this model. A detailed monitoring program would verify this model by detection of orbital motion of maser spots within the torus.

\section{Acknowledgments}

The authors gratefully acknowledge the VSOP, which is led by the Japanese Institute of Space and Astronautical
Science in cooperation with many organisations and radio telescopes around the world. We also acknowledge the VLBA, operated by the National Radio Astronomy Observatory, which is a facility of the National Science Foundation (NSF). This work was supported by Grant in Aid (c) 13640248 from the Japan Society for the Promotion of Science.

\section{References}

Bicknell, G. V., Dopita, M. A., \& O’Dea, C. P. 1997, ApJ, 485, 112 Claussen, M. J., Diamond, P. J., Braatz, J. A., Wilson, A. S., \& Henkel, C. 1998, ApJ, 500, L129

Guainazzi, M., \& Antonelli, L. A. 1999, MNRAS, 304, L15

Hirabayashi, H., et al. 1998, Science, 281, 1825

Jones, D. L., Wrobel, J. M., \& Shaffer, D. B. 1984, ApJ, 276, 480

Jones, D. L., Wehrle, A. W., Meier, D. L., \& Piner, B. G. 2000, ApJ, 534,165

Jones, D. L., Wehrle, A. W., Piner, B. G., \& Meier, D. L. 2001, ApJ, 553,968

Jones, D. L., et al. 1996, ApJ, 466, 63

Kadler, M., Ros, E., Zensus, J. A., Lobanov, A. P., \& Falcke, H. 2003, in SRT: The Impact of Large Antennas on Radio Astronomy and Space Science, SRT Conference Proceedings (Cagliari, Italy), Volume 1, in press (astro-ph/0204054)

Kameno, S., Sawada-Satoh, S., Inoue, M., Shen, Z.-Q., \& Wajima, K. 2001, PASJ, 53, 169

Kameno, S., Horiuchi, S., Shen, Z.-Q., Inoue, M., Kobayashi, H., Hirabayashi, H., \& Murata, Y. 2000, PASJ, 52, 209

Knapp, G. R., Gallagher, J. S., \& Faber, S. M. 1978, AJ, 83, 139

Marr, J. M., Taylor, G. B., \& Crawford, F. III 2001, ApJ, 550, 160

O’Dea, C. P. 1998, PASP, 110, 493

O’Dea, C. P., \& Baum, S. A. 1997, AJ, 113, 148

Peck, A. B., Taylor, G. B., \& Conway, J. E. 1999, ApJ, 521, 103

Scott, M. A., \& Readhead, A. C. S. 1977, MNRAS, 180, 539

Snellen, I. A. G., Schillizi, R. T., Miley, G. K., de Bruyn, A. G., Bremer, M. N., \& Röttgering, H. J. A. 2000, MNRAS, 319, 445

Stanghellini, C., O’Dea, C. P., Dallacasa, D., Baum, S. A., Fanti, R., \& Fanti, C. 1998, A\&AS, 131, 303

Sudou, H., Taniguchi, Y., Ohyama, Y., Kameno, S., SawadaSatoh, S., Inoue, M., Kaburaki, O., \& Sasao, T. 2000, PASJ, 52, 989

Tingay, S. J., \& Murphy, D. W. 2001, ApJ, 546, 210

Vermeulen, R. C., Readhead, A. C. C., \& Backer, D. C. 1994, ApJ, 430, L41

Vermeulen, R. C., Ros, E., Kellermann, K. I., Cohen, M. H., Zensus, J. A., \& van Langevelde, H. J. 2003, PASA, 20, 65

Walker, R. C., Romney, J. D., \& Benson, J. M. 1994, ApJ, 430, L45

Walker, R. C., Dhawan, V., Romney, J. D., Kellermann, K. I., \& Vermeulen, R. C. 2000, ApJ, 530, 233

Wrobel, J. M. 1984, ApJ, 284, 531

Wrobel, J. M., \& Heeschen, D. S. 1984, ApJ, 287, 41

Xiang, L., Stanghellini, C., Dallacasa, D., \& Haiyan, Z. 2002, A\&A, 385,768 